\title{
Alpha Oscillatory Activity Associated with Impaired Visual Search in Cerebral Visual Impairment
}

Christopher Bennett

Massachusetts Eye and Ear, Harvard Medical School

Corinna Bauer

Massachusetts Eye and Ear, Harvard Medical School

Peter Bex

Northeastern University

Davide Bottari

IMT Institute for Advanced Studies Lucca

LOTFI MERABET ( $\sim$ lotfi_merabet@meei.harvard.edu )

Massachusetts Eye and Ear, Harvard Medical School

\section{Research Article}

Keywords: CVI, EEG, Oscillatory, Visual

Posted Date: November 24th, 2020

DOI: https://doi.org/10.21203/rs.3.rs-107877/v1

License: (c) (1) This work is licensed under a Creative Commons Attribution 4.0 International License. Read Full License 


\section{Abstract}

Cerebral (cortical) visual impairment (CVI) is associated with perinatal damage to retrochiasmatic pathways and cerebral structures. Individuals with CVI often present with impairments in visual spatial processing and attention. However, the neural correlates associated with these higher order perceptual deficits remain unknown. In this study, we investigated visual search performance using a novel virtual reality based task combined with electroencephalography (EEG) and time frequency decomposition of recorded oscillatory activity. We found that CVI participants showed impaired visual search performance (indexed by both decreased success rates and increased reaction times) compared to age matched controls. Furthermore, analysis of captured EEG activity revealed that posterior alpha desynchronization (typically associated with coordinating neural activity in perceptual and cognitive tasks) was greatly impaired in CVI compared to control participants. Finally, exploratory analysis suggested a potential association between amplitude of oscillations in the alpha range and morphometric anatomical measures. Specifically, less alpha desynchronization following stimulus onset was correlated with decreased volume of specific thalamic nuclei. These results suggest that in the setting of early neurological damage to retrochiasmatic visual pathways, alpha desynchronization remains greatly impaired and may be related to deficits in visual spatial processing and attention commonly observed in this population.

\section{Introduction}

Cerebral visual impairment (CVl; also referred to as cortical or brain-based visual impairment) is the leading cause of pediatric congenital visual impairment in developed countries ${ }^{1}$. In contrast to visual impairment associated with pathology of the eye, CVI is defined as significant and verifiable visual dysfunction associated with damage to retrochiasmatic pathways and cerebral structures that cannot be attributed to disorders of the anterior visual pathways or potentially co-occurring ocular pathology 2,3 . There are numerous causes of $\mathrm{CVI}$, including perinatal hypoxia/ischemia, head injury/trauma, infection (e.g. encephalitis, meningitis), as well as genetic and metabolic disorders ${ }^{4}$. Early neurological injury is believed to dramatically alter the development of the brain including cortical gray matter, white matter tracts, and subcortical structures such as the thalamus ${ }^{5}$.

Children and adolescents with CVI exhibit a variety of visual deficits including decreased acuity and contrast sensitivity, as well as visual field restriction (typically in the lower hemi-field). In addition, many children with CVI present with higher order perceptual visual dysfunctions related to spatial processing and attention ${ }^{6-8}$ and highly variable visual functioning in response to increasing visual task demands and environmental complexity ${ }^{9}$. These visual impairments can profoundly impact a child's development, learning, mobility, and independence ${ }^{10}$.

The recording of visual evoked potentials (VEPs) in response to a variety of visual stimuli (e.g. flash, sweep, and patterns such as checkerboard or gratings) can be diagnostically useful, particularly in 
younger patients with CVI who cannot perform standard visual acuity testing (see ${ }^{11}$ for review and further discussion). However, disparities between assessments of grating visual acuity obtained by preferential looking compared to VEP acuity have been reported ${ }^{12}$. There are also limitations associated with traditional approaches to VEP recordings. For example, the visual stimuli and tasks employed are typically simplistic and thus do not necessarily reflect the true extent of behavioral visual processing deficits related to viewing complex and naturalistic visual scenes. Finally, the analysis of VEP waveform properties is also limited to the evaluation of neural activity that is phase locked to stimulus onset. Thus, there remains a need to not only develop novel assessment tools that can characterize functional visual performance using ecologically valid stimuli and tasks (i.e. with high behavioral relevance) ${ }^{13}$, but also more advanced electroencephalogram (EEG) based analysis to fully characterize neural activity linked with higher order visual processing deficits associated with CVI. Regarding this latter issue, neural oscillations represent how information is encoded, transferred, and integrated between distinct brain regions across multiple temporal scales ${ }^{14}$. They comprise both the evoked activity (phase-locked to stimulus onset) and the induced activity (non-phase-locked to stimulus onset, showing trial-to-trial variations in latency) ${ }^{15-17}$.

The human EEG is characterized by several prominent and important frequency band signals. Notably, posterior alpha oscillatory activity (within the 8 to $12 \mathrm{~Hz}$ band) reflects excitatory/inhibitory balance within the visual system which has been associated with coordinating neural activity in perceptual and cognitive tasks ${ }^{18,19}$. Alpha synchronization (i.e. an increase in alpha activity) results in an inhibition of task-irrelevant neural circuits while alpha desynchronization (i.e. a decrease in alpha activity) results from the engagement of task relevant neural processing ${ }^{18,19}$. Previous studies have suggested that alpha activity is highly dependent on structured visual experience occurring early in development and thus, represents an important marker of functional brain development. For example, EEG recordings from human newborn infants revealed that alpha activity is markedly reduced and shows a developmental time course that normalizes during late childhood ${ }^{20}$. Alpha activity has also been found to be dramatically reduced in adult individuals born with profound ocular blindness ${ }^{21}$. Finally, recent work in humans who underwent sight restoration surgery after a period of congenital blindness (i.e. removal of bilateral dense cataracts) has shown that alpha activity is not restored to levels comparable with neurotypical development ${ }^{22,23}$. The characterization of alpha activity in the case of congenital ocular based visual deprivation has provided important information regarding the neurophysiological basis of cortical oscillatory activity in relation to development and neuroplasticity. However, the impact of early damage to cerebral (i.e. retro-geniculate) visual pathways and structures on these signals remains largely unknown. Thus, characterizing alpha activity in CVI may not only help uncover the neurophysiological basis of visual perceptual deficits in this population, but also represents a unique opportunity to investigate developmental vulnerability and neuroplasticity in relation to early damage to central visual processing pathways.

To address these gaps in our understanding, we have developed a combined behavioral-EEG paradigm that incorporates a visual search task using a virtual reality (VR)-based naturalistic environment 
combined with electrophysiological recordings, with a particular focus on characterizing alpha activity in relation to behavioral performance. Visual search serves as an important paradigm to explore the deployment of visual attention, spatial processing, and characterizing the perceptual limits of the human visual system ${ }^{24}$. The use of a VR-based visual search task allows for the characterization of functional visual performance using an ecologically valid stimulus (i.e. with high behavioral relevance) that more closely approximates a real-world and behaviorally relevant task while maintaining a high level of engagement and motivation for participants as well as experimental control.

We hypothesized that individuals with CVI would show impaired visual search performance compared to age matched controls with neurotypical development. We further hypothesized that visual processing performance in CVI would be associated with differences in alpha activity, consistent with growing evidence that oscillatory activity in this frequency band represents an important marker of functional brain development. Finally, we explored possible connections between the amplitude of oscillations in the alpha range and standard clinical measures (i.e. visual acuity), behavioral performance, and subcortical anatomical markers of early neurological injury.

\section{Results}

\section{Behavioral Task Performance}

Figure $1 \mathrm{~A}$ shows success rates for the $\mathrm{CVI}$ and control groups across task difficulty conditions (low and high). Mean success rates for CVI (overall: $84.9 \% \pm 18.8$ SD, low condition: $86.6 \% \pm 17.71$ SD, high condition: $83.2 \% \pm 20.9$ SD) were lower compared to controls (overall: $98.1 \% \pm 3.9$ SD, low condition: 99.0\% \pm 2.4 SD, high condition: $97.2 \% \pm 5.0 \mathrm{SD}$ ). A 2-way ANOVA (factors: group $x$ condition) revealed a statistically significant difference for group $\left[F(1,18)=10.774, p=0.002, \eta_{p}^{2}=0.230\right]$. While there was an overall trend for worsening performance as a function of task difficulty condition in both groups, this did not reach statistical significance $\left[F(1,18)=0.548, p=0.464, \eta_{p}^{2}=0.015\right]$. The test for an interaction effect was not found to be statistically significant $\left[F(1,36)=0.093, p=0.762, n_{p}{ }^{2}=0.003\right]$.

Reaction time data for the CVI and control groups and across task difficulty conditions are shown in figure $1 \mathrm{~B}$. Mean reaction times for the CVI group (overall: $1.478 \mathrm{sec} \pm 0.694 \mathrm{SD}$, low condition: $1.467 \mathrm{sec}$ $\pm 0.738 \mathrm{SD}$, high condition: $1.490 \mathrm{sec} \pm 0.647 \mathrm{SD}$ ) were higher than controls (overall: $1.101 \mathrm{sec} \pm 0.392$, low condition: $1.056 \mathrm{sec} \pm 0.383 \mathrm{SD}$, high condition: $1.147 \mathrm{sec} \pm 0.402 \mathrm{SD}$ ). A 2-way ANOVA (factors: group $x$ condition) revealed a statistically significant difference for group $\left[F(1,18)=79.314, p<0.001, \eta_{p}{ }^{2}\right.$ $=0.109]$, but not for the task difficulty condition $\left[F(1,18)=1.725, p=0.190, \eta_{p}{ }^{2}=0.003\right]$. The test for an interaction effect was not statistically significant $\left[F(1,36)=0.595, p=0.441, \eta_{p}{ }^{2}=0.001\right]$.

Finally, analysis of off-screen data (serving as an index measure of testing compliance; see Methods) from control and CVI participants revealed no statistical difference between the two groups throughout 
the visual search task (control mean $=15.182 \pm 12.194 \mathrm{SD}$ and $\mathrm{CVI}$ mean $=16.380 \pm 32.513 \mathrm{SD} ; \mathrm{t}=$ $0.689, p=0.491)$.

\section{EEG Analysis}

Time frequency decomposition of the EEG data in controls and across task difficulty conditions are shown in figure $2 \mathrm{~A}$. Visual inspection revealed a strong and well-defined alpha desynchronization signal for both conditions with maximal desynchronization occurring between 0.5 and 0.7 sec post stimulus onset. Within the control group, cluster-based permutation analysis revealed a significant difference between the low compared to high task difficulty conditions (negative cluster, $p=0.005$ ). This significant effect was found primarily in the alpha $(8-12 \mathrm{~Hz})$ frequency range peaking around 0.5 to $0.6 \mathrm{sec}$ after stimulus onset, and mostly involved electrodes around the occipital region. In particular, there was greater negative power in the alpha band for the low compared to high task difficulty condition, and this difference was statistically significant $[\mathrm{t}(11)=3.651, \mathrm{p}=0.004]$. This result suggests that in controls, alpha desynchronization was modulated by task difficulty.

The response profile in $\mathrm{CVI}$ subjects appeared to be altered across low-range frequencies, but particularly in the alpha range. Of note, there was a distinct lack of a strong and well-defined alpha desynchronization responses for both task difficulty conditions. While there were significant condition differences for controls, the cluster permutation analysis revealed no significant clusters between the low and high task difficulty conditions within the CVI group (all cluster p's $>0.281$ ). This result suggests a lack of alpha desynchronization modulation in CVI based on task difficulty.

In our first between group statistical comparison, we tested differences at the low and high task difficulty conditions. The cluster-based permutation analysis revealed a significant between group difference for low (negative cluster, $p=0.001$, see Figure $2 B$ ) and for high (negative clusters, $p=0.002$ and $p=0.036$, see Figure $2 \mathrm{~B}$ ) conditions. The significant effect for the low condition mostly involved electrodes around the occipital region, was found primarily in the alpha $(8-12 \mathrm{~Hz})$ frequency range, and peaked between 0.440 to $0.500 \mathrm{sec}$ after stimulus onset (see Figure $2 \mathrm{~B}$ and $2 \mathrm{D}$ ). The significant effect for the high task difficulty condition involved a broader range of occipital and frontal electrodes, was found across a larger frequency-band range including alpha and extending to beta $(8-25 \mathrm{~Hz})$ range, and with the peak statistical difference observed between 0.500 and $0.560 \mathrm{sec}$ after stimulus onset (see Figure $2 \mathrm{~B}$ and 2 D). Specific group comparisons in the alpha range revealed a statistically significant difference for both the low $[\mathrm{t}(18)=-4.613, \mathrm{p}=0.002]$ and high $[\mathrm{t}(11)=-2.325, \mathrm{p}=0.032]$ task difficulty conditions. Scalp topographies for the two groups' alpha activity and for the two task conditions are displayed in figure 2 (scalp topographies comprise the activity measured within a $60 \mathrm{msec}$ time-window including maximal statistical difference; 0.440 to $0.500 \mathrm{sec}$ for low and 0.500 to $0.560 \mathrm{sec}$ for high conditions; figure $2 \mathrm{C}$ ).

Separate comparisons for each group and condition of alpha oscillations to 0 revealed significant alpha desynchronization for controls [low: $\mathrm{t}(11)=-6.238, \mathrm{p}=0.001$; high: $\mathrm{t}(11)=-3.046, \mathrm{p}=0.011$ ], but not for the CVI group [low: $t(7)=1.438, p=0.194$; high: $t(7)=1.260, p=0.248]$. This is consistent with a lack of alpha desynchronization in the CVI group during the post-stimulus presentation window. 
Finally, an examination of potential group differences in alpha oscillations was conducted to determine whether the two groups differed prior to stimulus onset and whether this could potentially have an influence on post stimulus alpha oscillatory activity. A statistical analysis was carried out for the time window between -0.6 to $-0.1 \mathrm{sec}$ prior to stimulus onset. No statistically significant differences were found between $\mathrm{CVI}$ and control participants within this pre-stimulus window $(\mathrm{p}>0.100)$. This finding suggests that only the alpha oscillatory activity occurring after stimulus onset was affected in CVI.

\section{Correlations Between A/pha Oscillatory Activity and Behavioral and Morphometric Measures}

We conducted an exploratory analysis within the CVI group to investigate possible functional and structural associations between the level of alpha desynchronization signal and clinical, behavioral, and morphometric measures of interest.

Relating alpha desynchronization (mean EEG amplitude at 8 to $12 \mathrm{~Hz}$ from time 0.500 to $0.700 \mathrm{sec}$ ) and visual acuity (based on the logMAR value of the better seeing eye) revealed no significant correlation $\left(r^{2}=\right.$ $0.001, p=0.951)$. Secondly, associating alpha desynchronization and behavioral performance also did not reveal a significant statistical correlation (success rate: $r^{2}=0.001, p=0.983$; reaction time: $r^{2}=0.028$, $p=0.365)$.

Finally, we examined possible links between alpha desynchronization and the volume of thalamic nuclei (see Supplementary Figure $1 \mathrm{~A}$ ). Results of this analysis suggest significant negative correlations between alpha desynchronization levels and the volumes of two nuclei. Specifically, these were the left ventral lateral anterior $\left(r^{2}=0.69 ; p=0.042\right)$ and left lateral pulvinar $\left(r^{2}=0.69 ; p=0.042\right)$ nuclei. These results suggest that that lower thalamic nuclei volumes were associated with more positive alpha desynchronization values.

\section{Discussion}

The present work discusses results of a combined behavioral-EEG paradigm comparing individuals with $\mathrm{CVI}$ and control subjects with neurotypical development. Specifically, we compared visual search performance on a VR-based, ecologically valid task in relation to time frequency decomposition of recorded oscillatory activity with a particular focus on alpha desynchronization levels.

Regarding behavioral results, we found that overall, CVI participants showed impaired visual search performance compared to controls. Specifically, success rates were lower and reaction time measures were longer for $\mathrm{CVI}$ subjects. We interpret these findings as suggesting that for this VR-based visual search task, individuals with CVI were more likely to miss and took longer time to fixate the correct target. These observations are consistent with clinical observations in this population relating to impairments with visual spatial processing and attention ${ }^{6-8}$. Furthermore, these differences in behavioral results cannot be explained by poor testing compliance on the part of the CVI participants given that there were no significant group differences in terms of captured off-screen data. 
EEG responses to the visual search task revealed marked differences between the CVI and control groups with respect to oscillatory activity, with the response profile in CVI subjects appearing to be impaired across frequencies. More specifically, there was a distinct lack of a strong and well-defined alpha desynchronization response in participants with $\mathrm{CVI}$ compared to controls for both task difficulty conditions. Furthermore, we found that while differences in the level of alpha desynchronization with respect to task difficulty were significant in controls, this modulation was not found in the CVI group. Overall, these group differences in alpha desynchronization levels and modulation with respect to task difficulty appear to be related to differences in behavioral performance between $\mathrm{CVI}$ and control participants. We interpret these findings to suggest that in CVI, there is an impairment in the typical development and recruitment of visual sensory and attentional systems necessary to carry out this behavioral task. We also noted that these differences in alpha desynchronization were found to be limited to post-stimulus signal changes, as no significant differences in pre-stimulus alpha signal were observed between the two groups. This finding may serve as an indication that differences in alpha desynchronization are largely driven by the modulation of visual system activity following the onset of the visual stimulus, rather than differences in baseline activity or preparatory brain responses. Taken together, these results suggest that the establishment of alpha desynchronization and its functional modulation during visual processing is dramatically impaired in CVI. Finally, exploration of potential correlations between alpha oscillatory activity and clinical, behavioral, and anatomical measures of interest in the CVI group revealed that alpha desynchronization levels were not significantly correlated with visual acuity or visual search performance (i.e. success rate and reaction times). However, analysis of EEG signals with respect to thalamic volume showed a significant association between more positive alpha desynchronization values and lower volumes of certain thalamic nuclei. Specifically, these were within the left ventral lateral and left lateral pulvinar nuclei which are regions known to be involved with the initiation and control of saccadic eye movements as well as regulation of visual attention ${ }^{25}$. Given the purported role of these thalamic nuclei, it is possible that their reduced integrity in CVI may also be associated with impaired visual search performance in this group.

It is important to note that characterization of higher order visual perceptual deficits in CVI remains crucial, as these may otherwise go undetected using standard clinical assessments such as visual acuity. Indeed, mounting evidence suggests that impaired spatial processing appears to be a common consequence of neurodevelopmental damage ${ }^{26}$ and represents the most common type of visual impairment observed in children with $\mathrm{CVI}^{27}$. It is suspected that this "dorsal stream dysfunction" is associated with the inherent vulnerability of regions within the posterior parietal lobes that are highly susceptible to early developmental injury ${ }^{27}$. Previous psychophysical studies in children born premature and with developmental delays have characterized visual spatial processing impairments using a variety of stimuli including biological form from motion, optic flow fields, and spatial integration tasks (e.g. ${ }^{28-}$ ${ }^{30}$ ). While these visual stimuli may offer excellent control of various testing parameters (e.g. isolating stimulus factors), they remain limited in terms of their ecological validity and behavioral relevance. In other words, it is difficult to draw inferences and translate results obtained from these types of psychophysical test stimuli with respect to how individuals with CVI interact with their visual 
surroundings. To address this concern, some groups have incorporated a series of desktop computerized tasks combined with eye tracking metrics to assess ocular motor functions and higher order visual processing abilities in CVI. Using moving cartoon images, Kooiker and colleagues were able to demonstrate clear deficits related to visual search, fixation and ocular motor pursuit in children with CVI 31,32. In this study, we further expanded on the importance of characterizing visual search performance by employing a VR-based task requiring participants to look for a target toy placed within an array of other toys, and at two levels of task difficulty. This novel approach allowed for characterization of functional visual performance using a naturalistic, engaging, and behaviorally relevant task. Furthermore, the inherent design of this VR-based task recruits skills related to visual search and attention, thus allowing for the characterization of behavioral performance typically ascribed to the dorsal visual processing stream ${ }^{33}$.

Previous studies have analyzed electrophysiological recordings in response to a variety of visual stimuli (e.g. flash, sweep, and patterns such as checkerboard or gratings) in an attempt to identify neural correlates associated with visual impairments in CVI ${ }^{34-36}$. In one study, visual motion processing in CVI was investigated using steady state visual evoked potentials (ssVEP) and compared patterns of occipital activation in response to viewing local and global motion stimuli ${ }^{30}$. It was found that CVI subjects showed selective global (but not local) motion deficits, especially for slower stimulus velocities, with corresponding greater decreases in occipital signal amplitudes ${ }^{30}$. In a more recent study, verMass and colleagues (2020) used magnetoencephalography (MEG) to investigate potential differences in oscillatory activity while viewing a high contrast spatial grating stimulus in a cohort of children with cerebral palsy (CP); a neurodevelopmental disorder which often presents with CVI. The authors found that viewing visual gratings induced a decrease in alpha-beta $(10-20 \mathrm{~Hz})$ activity, and an increase in both low $(40-56 \mathrm{~Hz})$ and high $(60-72 \mathrm{~Hz})$ gamma oscillations in the occipital cortex ${ }^{37}$. Furthermore, the strength of the frequency specific cortical oscillations were significantly weaker in the children with $\mathrm{CP}$ consistent with impaired visual processing deficits as compared to controls ${ }^{37}$. Our results appear to be consistent with these findings of decreased alpha activity in response to visual processing, and further extend these observations to higher order processing related to visual search performance and signal modulation with respect to task difficulty.

As mentioned earlier, previous work from human newborn infants ${ }^{20}$, individuals with congenital and profound ocular blindness ${ }^{21}$, as well as sight restoration surgery (i.e. removal of dense bilateral cataracts) 22,23 , have provided converging evidence that alpha activity is highly dependent on structured visual experience occurring early in development. The results of our study expand upon the notion that this signal represents an important marker of functional brain development. In the case of CVI, which has been defined as a condition associated with perinatal damage to retrochiasmatic pathways and cerebral structures $2,3,27$, we found that the establishment of alpha desynchronization signals occurring during visual processing was dramatically impaired. It is also worth noting that this was similarly the case in CVI participants with normal (or near normal) visual acuity. Thus, even in the case of structured visual experience occurring early in development, damage to central visual processing areas appears to 
dramatically alter the formation of appropriate visual cortical oscillatory activity which in turn, may be associated with higher order visual processing deficits observed in the CVI population.

Finally, though still preliminary, correlation analyses suggest that there may be a link between early thalamic injury and the level of alpha desynchronization signals. Specifically, we found that lower thalamic nuclei volumes (i.e. the left ventral lateral anterior and left lateral pulvinar) were associated with more positive alpha desynchronization values. This finding may not be surprising given the purported role of these thalamic nuclei in the initiation and control of saccadic eye movements as well as regulation of visual attention ${ }^{25}$. However, these results should be interpreted with caution and further studies exploring possible correlations between thalamic damage and alpha oscillatory activity are needed in order to confirm these preliminary observations. It is important to highlight that while the origins of alpha oscillations remain debated, these preliminary findings may also provide cues as to the relative contribution and importance of thalamic integrity with respect to establishing alpha desynchronization activity in humans ${ }^{38}$. While there is evidence that infragranular layers of the cortex generate alpha rhythm ${ }^{39-42}$, several studies have revealed that cortical alpha activity occurs coherently with thalamic activity 43,44 . Supporting this view, evidence from work in non-human primates suggests that the alpha rhythm generated by the pulvinar is associated with attentional processing occurring at the cortical level 45 .

The major limitation of the present study relates to the relatively small study size sample. It is worth mentioning however that despite this limitation, we did observe a very large difference between CVI and control participants in terms of alpha desynchronization levels in response to our visual search task (Cohen's $\mathrm{d}$ : 1.25 ; effect size $r=0.53$ ). This suggests that in the setting of early damage to retrochiasmatic pathways and cerebral structures (as in the case of $\mathrm{CVI}$ ), the development of alpha desynchronization is dramatically impaired and further speaks to the importance of this signal as a marker of functional brain development.

The variable causes of CVI (as well as the timing of injury) can create a heterogeneous study population, thus making it more difficult to reveal links between behavioral-EEG responses and the nature of the neurological injury. Future studies with larger sample sizes and greater representations of the various etiologies of $\mathrm{CVI}$ will be needed to better characterize visual processing deficits and the profile of EEG responses with respect to the cause and timing of neurological injury associated with this condition.

In general, the recruitment of participants with $\mathrm{CVI}$ who can carry out the demands related to combined behavioral-EEG studies remains challenging. However, the design of the current study methodology does present important advantages. As mentioned previously, the use of an ecologically valid, behaviorally relevant visual search task is likely to help better characterize visual processing deficits associated with $\mathrm{CVI}$ under more naturalistic viewing conditions. Furthermore, the use of a wireless EEG recording montage allows for greater flexibility and is more amenable for longer periods of signal capture especially when working with children and adolescents with developmental disabilities. 
Finally, while the current study focused on analyzing oscillatory activity in a low frequency range (3 - 40 $\mathrm{Hz}$ ) and most notably alpha desynchronization, future work will investigate potential differences in other frequency bands. In particular, the analysis of gamma oscillatory activity should also be considered as activity in this frequency band have been associated with low-level sensory processing, higher-order visual spatial processing, and complexity related to spatial or visual tasks in humans ${ }^{17,46}$. Further studies will also consider the analysis of EEG responses in other cortical foci of interest using higher density electrode montages (and with a particular focus on areas associated with the dorsal visual processing stream) in order to further characterize the neurophysiological basis of visual processing deficits in CVI.

\section{Methods}

\section{Participants}

Eight individuals with clinically diagnosed $\mathrm{CVI}$ and aged between 15 and 22 years ( 3 female, mean age: 18.0 years old $\pm 2.67 \mathrm{SD}$ ) participated in the study. All participants in the CVI group possessed a sufficient level of visual acuity to complete the behavioral task (ranging from 20/20 to 20/60 Snellen equivalent in the better seeing eye). A full list of participant details can be found in Table 1. Twelve individuals with neurotypical development and aged between 14 and 25 years ( 5 female, mean age: 19.92 years old \pm $2.61 \mathrm{SD}$ ) served as comparative controls. Control participants had normal or corrected to normal visual acuity and no previous history of any ophthalmic (e.g. strabismus, amblyopia) or neurodevelopmental (e.g. epilepsy, attention deficit disorder) conditions. The groups were not statistically different with respect to mean age $(p=0.1283)$. All components of the study were approved by the investigative review board of the Massachusetts Eye and Ear, Boston MA, USA and all methods were performed in accordance with the relevant guidelines and regulations. Written informed consent was obtained from all the participants and a parent and/or legal guardian (in the case of a minor) prior to commencing the study. Informed consent to publish identifying images was also obtained prior to the preparation of this manuscript.

\section{Behavioral Task and Visual Stimulus Design}

We developed a VR based visual search task referred to as the "virtual toy box" (complete details regarding the overall design of the task can be found in ${ }^{47}$ ). Briefly, the behavioral task represents a rendering of a toy box with a $5 \times 5$ array of static toys presented in canonical view presented in a trial by trial fashion, and viewed from an overhead, first-person perspective. Participants were instructed to search, locate, and fixate a specific target toy (chosen from 3 options prior to data collection; a blue truck, orange basketball, and yellow duck) placed among surrounding distracting toys and without overlap. Target selection was incorporated in order to enhance the immersive feel of the task and confirm that the participant was able to correctly identify the target toy in isolation before commencing the task. The visual scene was developed using the Unity 3D game engine version 5.6 (Unity Technologies) and on an Alienware Aurora R6 desktop computer (Intel i5 processor, NVidia GTX 1060 graphics card, and 32 GB of 
RAM; Alienware Corporation). 3D object models were created in house using Blender modeling software (Blender Foundation). The visual task was run using Presentation software (https://www.neurobs.com/) to control simultaneous EEG event markers and signal recording.

Based on preliminary work ${ }^{47}$, we selected two condition levels in order to explore potential differences in behavioral performance and recorded EEG signal as a function of task difficulty. In the "low" task difficulty condition, the target toy was surrounded by 1 unique distractor toy and background clutter was not present (figure $3 \mathrm{~A}$ ). In the "high" task difficulty condition, 9 unique distractor toys were presented alongside the target toy, and a cluttered background scene was also present (figure $3 \mathrm{~B}$ ).

A trial consisted of 2 secs of viewing of the toy box scene followed by $1 \mathrm{sec}$ of a blank gray screen with a fixation cross. This was repeated 50 times per run, with a total of 4 runs collected for a total of 200 trials. An equal number of low and high task difficulty trials were presented and in a pseudorandom order. Each run lasted 2.5 mins with a brief rest period given between each run.

Participants were seated comfortably $60 \mathrm{~cm}$ in front of a 27" LED monitor (ViewSonic 27" Widescreen 1080p; 1920x1080 resolution) (figure $3 \mathrm{C})$. Visual search patterns ( $X, Y$ coordinate positions of gaze on the screen) were captured under binocular viewing conditions using the Tobii 4C Eye Tracker system (90 $\mathrm{Hz}$ sampling rate; Tobii $A B$ ). Prior to the first experimental run, eye tracking calibration was performed for each participant (Tobii Eye Tracking Software, v 2.9 calibration protocol) which took less than one minute to complete. The process included a 7 point calibration task (screen positions: top-left, top-center, topright, bottom-left, bottom-center, bottom-right, and center-center) followed by a 9 point post calibration verification (same 7 calibration points plus center-left and center-right). Accuracy criterion was determined by gaze fixation falling within a $2.25^{\circ}$ (arc degree) radius around each of the 9 points and further confirmed by visual inspection prior to commencing data collection.

\section{Behavioral Data Capture, Analysis, and Outcome Measures}

Eye tracking data characterizing visual search performance and responses were captured as participants initially searched, located, and then fixated the target on the screen. Three measures were used to objectively quantify visual search performance based on the collected eye gaze data. Success rate for correctly locating the target was determined based on whether a participant was able to fixate the target on a given trial. Fixation was defined as gaze that remained within the outer contour of the target for a minimum of $0.400 \mathrm{sec}$. A percentage was then calculated for overall performance as well as for the two task difficulty conditions (low and high). Reaction time was defined as the first moment the participant's gaze arrived within the outer contour of the target and fixated the target on the screen for a given trial. Finally, given the position of the participant's gaze on the screen was constantly recorded, we also determined how often/long they were looking at and away from the screen on a given trial. This offscreen data capture represents the number of gaze points per trial that fall outside of the bounds of the screen and thus serve as an index measure of test compliance and reliability. 
A mixed-model ANOVA was conducted separately for the primary behavioral measures (success rate and reaction time). Each ANOVA included the between-factor of group and the within-factor of task difficulty condition. Post-hoc Bonferroni corrected t-test comparisons were conducted for significant main effects. In a subset of CVI participants, a Spearman rank correlation was used to investigate potential associations between levels of alpha desynchronization and clinical, behavioral, and anatomical measures of interest (i.e. visual acuity, success rate, reaction time, and thalamic nuclei volume). Statistical analyses were performed using SAS University Edition statistical package.

\section{EEG Task, Software, and Montage}

A wireless 20-channel Enobio system (sampling rate of $500 \mathrm{~Hz}$; Neuroelectrics, Barcelona, Spain) was used to collect EEG data and feeding to a wireless Bluetooth transmitter. Solid gel electrodes were used and placement corresponded to the standard 10-20 international system (with the reference channel connected to an ear clip placed on the participant's right ear lobe).

Signals were captured on-line and recorded by software running on a desktop located within $1 \mathrm{~m}$ of the test participant. Once the system was fully set up, the quality of signal from each channel was verified. If signal noise was present in any of the channels, the electrode was checked and additional conductive gel was added to ensure adequate signal recording quality. After final checks were completed, the participant would begin the study.

\section{EEG ProcessingPipeline and Analysis Strategy.}

The analysis was performed by implementing a corroborated pipeline ${ }^{48,49}$. Raw EEG data were preprocessed with EEGLAB 13.6.5 $b^{50}$. First, a low pass filter (windowed sinc FIR filter, cut-off frequency 40 $\mathrm{Hz}$, filter order 500 ) as well as a high pass filter (windowed sinc FIR filter, cut-off frequency $1 \mathrm{~Hz}$, filter order 100; ${ }^{51}$ were applied. Data were resampled to $250 \mathrm{~Hz}$. In order to remove non-stereotypical artefacts (such as sudden increases of muscle activity) from the data, continuous datasets were segmented into consecutive epochs with a length of $1 \mathrm{sec}$. Segments displaying a joint probability of activity ${ }^{52}$ larger than three standard deviations (SD) were removed before performing the independent component analysis (ICA). To remove typical artefacts such as eye movements and eye blinks, an ICA based on the extended Infomax ${ }^{53-55}$ was performed. In order to reduce computational time, the number of decomposed components was reduced from 20 to 15. The ICA weights were then associated to the raw EEG (continuous and unfiltered data). ICA components representing artefacts were identified with the semi-automatic algorithm CORRMAP ${ }^{56}$. Following artefact-related component removal the data were segmented accordingly to stimulus presentation. The data were segmented into 3 seconds epochs (- 1 to $2 \mathrm{sec}$; full behavioral trial length plus rest). Epochs having a joint probability of activity greater than three standard deviations were automatically rejected. This led to at least $88 \%$ of artifact free trials remaining per participant to be used in subsequent time-frequency analysis. Electrophysiological data were subsequently analyzed using the FieldTrip software ${ }^{57}$. 
Epoched data were analyzed performing a time-frequency decomposition. Time-frequency transformation was computed at each channel by convolving the data with a complex Morlet wavelet $(\mathrm{t}, \mathrm{f0})$ which had a Gaussian shape in time $(\sigma t)$ and in frequency

(of) around the center frequency (f0). Non-constant wavelets increasing from fo/ of $=4$ to 13 for frequencies from 3 to $40 \mathrm{~Hz}$ (step size $1 \mathrm{~Hz}$ ) were employed. Time-frequency analyses were conducted per channel using a wavelet transform, known to represent a balance between time and frequency resolution ${ }^{17,23}$. The wavelet transform was conducted for the entire epoch range (-1.000 to $\left.2.000 \mathrm{sec}\right)$ and was done in steps of $0.020 \mathrm{sec}$. The data transformation was performed before averaging across

trials, separately for each frequency band. The total power of each frequency band represents both the phase locked and the non-phase locked signal with respect to the event. The resulting power was baseline corrected to obtain the relative signal change: $\mathrm{P}(\mathrm{t}, \mathrm{f})$ corrected $=(\mathrm{P}(\mathrm{t}, \mathrm{f})$ post-stimulus$\mathrm{P}(\mathrm{f})$ baseline $) / \mathrm{P}(\mathrm{f})$ baseline. The pre-stimulus period between -700 to $-300 \mathrm{~ms}$ served as baseline for all spectral analyses. This baseline prevented including eventual slow frequency leakage occurring immediately before the stimulus onset

The first statistical comparison between groups was done using parametric cluster-based permutation tests ${ }^{58}$ at each condition level (low and high). This approach identifies clusters of significant effects in time, frequency and space, and controls for multiple comparisons. Cluster-based permutation analysis was run without a bias from any priori assumptions about specific frequency bands, regions of interest (ROI) or time intervals. Thus, across all channels, frequency range [3-40Hz], and time-window $[0-1 \mathrm{sec}]$ after stimulus presentation. We used ft_freqstatistics function implemented in FieldTrip (Monte Carlo sampling method, 5,000 iterations, cluster alpha $=0.05$, maxsum criterion, minimum spatial extent $=1$ channel). Identified clusters were considered as being significant at $p<0.025$.

At the group level, baseline-corrected time-frequency representations were computed using a cluster of occipital electrodes $\left(\mathrm{O}_{1}, \mathrm{O}_{\mathrm{z}}\right.$, and $\left.\mathrm{O}_{2}\right)$ for each condition and group (shown in figure $2 \mathrm{~A}$ ). 2-D topographical maps of baseline-corrected data were also computed specifically in the peak alpha desynchronization time window ( 0.440 to $0.500 \mathrm{sec}$ for low and 0.500 to $0.560 \mathrm{sec}$ for high). Furthermore, alpha desynchronization was statistically compared within the post-stimulus peak window $(0.5$ to $0.7 \mathrm{sec})$ using paired-sample t-tests for within group comparisons and independent sample t-tests for between group comparisons. Pre-alpha desynchronization was also statistically compared within an extracted prestimulus window $(-0.6$ to $-0.1 \mathrm{sec})$ using an independent sample t-test between groups (note that there was no pre-stimulus indication for a given condition, therefore group data were averaged across conditions).

\section{Determination of Morphometry Volume of Thalamic Nuclei}

Morphometry data was available from a subset of participants with CVI $(n=6)$ who had previously participated in an MRI scanning protocol. Specifically, two $\mathrm{T}_{1}$-weighted anatomical scans (TE $3.1 \mathrm{~ms}$, TR $6.8 \mathrm{~ms}$, flip angle $9^{\circ}$, isotropic $1 \mathrm{~mm}$ voxel size) were acquired with an 8-channel phased array head coil 
(Philips 3T Intera Achieva). The volume of predetermined thalamic nuclei was quantified for each subject in anatomical space in FreeSurfer (https://surfer.nmr.mgh.harvard.edu/) ${ }^{59-62}$. Briefly, the two consecutive $\mathrm{T}_{1}$-weighted volumes were co-registered and averaged to improve the signal-to-noise ratio prior to skull stripping, intensity normalization, and Talairach registration. To evaluate specific thalamic nuclei within each participant, 26 thalamic nuclei were isolated in each subject's anatomical space from a probabilistic Morel histological atlas ${ }^{63-65}$ (see Supplementary Figure 1). Residuals were used to remove the effects of intracranial volume on the volume of each segmented thalamic nucleus. The thalamic parcellations show excellent test-retest reliability, good agreeability with stereology, and are robust to abnormal brain morphology 63,64 .

\section{Declarations}

\section{Acknowledgements}

This work was supported by grants from the NIH/NEI (R21 EY030587 to LBM and R01 EY030877 to $\mathrm{CMB}$ ), the Massachusetts Lions Eye Research Fund to LBM and CMB, and Deborah Munroe Noonan Memorial Research Fund to LBM. The authors also thank Emma Bailin and Timothy Gottlieb for assistance in developing some of the task stimuli, as well as the participants and their families for their willingness to participate in this study.

\section{Author Contributions}

All authors contributed to the design, analysis of results, preparation of this manuscript.

\section{Competing Interests}

The authors declare no competing interests

\section{Data Availability}

The datasets generated during and/or analyzed during the current study are available from the corresponding author on reasonable request.

\section{References}

1. Solebo, A. L., Teoh, L. \& Rahi, J. Epidemiology of blindness in children. Arch Dis Child102, 853-857, doi:10.1136/archdischild-2016-310532 (2017).

2. Dutton, G. N. Cognitive vision, its disorders and differential diagnosis in adults and children: knowing where and what things are. Eye (Lond)17, 289-304, doi:10.1038/sj.eye.6700344 (2003).

3. Sakki, H. E. A., Dale, N. J., Sargent, J., Perez-Roche, T. \& Bowman, R. Is there consensus in defining childhood cerebral visual impairment? A systematic review of terminology and definitions. $\mathrm{Br} J$ Ophthalmo/102, 424-432, doi:10.1136/bjophthalmol-2017-310694 (2018). 
4. Hoyt, C. S. Visual function in the brain-damaged child. Eye17, 369-384, doi:10.1038/sj.eye.6700364 (2003).

5. Volpe, J. J. Brain injury in premature infants: a complex amalgam of destructive and developmental disturbances. Lancet Neuro/8, 110-124, doi:10.1016/S1474-4422(08)70294-1 (2009).

6. Dutton, G. N., McKillop, E. C. \& Saidkasimova, S. Visual problems as a result of brain damage in children. The British journal of ophthalmology90, 932-933, doi:10.1136/bjo.2006.095349 (2006).

7. Fazzi, E. et al. Cognitive visual dysfunctions in preterm children with periventricular leukomalacia. Dev Med Child Neuro/51, 974-981, doi:10.1111/j.1469-8749.2009.03272.x (2009).

8. Zihl, J. \& Dutton, G. H. in Cerebral Visual Impairment in Children: Visuoperceptive and Visuocogntive Disorders (ed G.N. Dutton J. Zihl) Ch. 4, 61-115 (Springer-Verlag Wien, 2015).

9. Jan, J. E., Groenveld, M., Sykanda, A. M. \& Hoyt, C. S. Behavioural characteristics of children with permanent cortical visual impairment. Dev Med Child Neuro/29, 571-576 (1987).

10. Merabet, L. B., Mayer, D. L., Bauer, C. M., Wright, D. \& Kran, B. S. Disentangling How the Brain is "Wired" in Cortical (Cerebral) Visual Impairment. Semin Pediatr Neurol24, 83-91, doi:10.1016/j.spen.2017.04.005 (2017).

11. Chang, M. Y. \& Borchert, M. S. Advances in the evaluation and management of cortical/cerebral visual impairment in children. Surv Ophthalmo/65, 708-724, doi:10.1016/j.survophthal.2020.03.001 (2020).

12. Lim, M. et al. Development of visual acuity in children with cerebral visual impairment. Arch Ophthalmo/123, 1215-1220, doi:10.1001/archopht.123.9.1215 (2005).

13. Parsons, T. D. Virtual Reality for Enhanced Ecological Validity and Experimental Control in the Clinical, Affective and Social Neurosciences. Front Hum Neurosci9, 660, doi:10.3389/fnhum.2015.00660 (2015).

14. Siegel, M., Donner, T. H. \& Engel, A. K. Spectral fingerprints of large-scale neuronal interactions. Nat Rev Neurosci13, 121-134, doi:10.1038/nrn3137 (2012).

15. David, O., Kilner, J. M. \& Friston, K. J. Mechanisms of evoked and induced responses in MEG/EEG. Neuroimage31, 1580-1591, doi:10.1016/j.neuroimage.2006.02.034 (2006).

16. Herrmann, C. S., Rach, S., Vosskuhl, J. \& Struber, D. Time-frequency analysis of event-related potentials: a brief tutorial. Brain Topogr27, 438-450, doi:10.1007/s10548-013-0327-5 (2014).

17. Tallon-Baudry, C. \& Bertrand, O. Oscillatory gamma activity in humans and its role in object representation. Trends in Cognitive Sciences3, 151-162 (1999).

18. Jensen, O., Bonnefond, M. \& VanRullen, R. An oscillatory mechanism for prioritizing salient unattended stimuli. Trends Cogn Sci16, 200-206, doi:10.1016/j.tics.2012.03.002 (2012).

19. Klimesch, W. alpha-band oscillations, attention, and controlled access to stored information. Trends Cogn Sci16, 606-617, doi:10.1016/j.tics.2012.10.007 (2012).

20. Eisermann, M., Kaminska, A., Moutard, M. L., Soufflet, C. \& Plouin, P. Normal EEG in childhood: from neonates to adolescents. Neurophysiol Clin43, 35-65, doi:10.1016/j.neucli.2012.09.091 (2013). 
21. Novikova, L. A. Blindness and the Electrical Activity of the Brain: Electroencephalographic Studies of the Effects of Sensory Impairment. American Foundation for the Blind Research Series23 (1973).

22. Bottari, D. et al. Motion processing after sight restoration: No competition between visual recovery and auditory compensation. Neuroimage167, 284-296, doi:10.1016/j.neuroimage.2017.11.050 (2018).

23. Bottari, D. et al. Sight restoration after congenital blindness does not reinstate alpha oscillatory activity in humans. Scientific reports6, 1-10 (2016).

24. Treisman, A. M. \& Gelade, G. A feature-integration theory of attention. Cogn Psycho/12, 97-136, doi:10.1016/0010-0285(80)90005-5 (1980).

25. Dominguez-Vargas, A. U., Schneider, L., Wilke, M. \& Kagan, I. Electrical Microstimulation of the Pulvinar Biases Saccade Choices and Reaction Times in a Time-Dependent Manner. J Neurosci37, 2234-2257, doi:10.1523/JNEUROSCI.1984-16.2016 (2017).

26. Johnston, R., Pitchford, N. J., Roach, N. W. \& Ledgeway, T. New insights into the role of motion and form vision in neurodevelopmental disorders. Neurosci Biobehav Rev83, 32-45, doi:10.1016/j.neubiorev.2017.09.031 (2017).

27. Dutton, G. N., Chokron, S., Little, S. \& McDowell, N. Posterior parietal visual dysfunction: An exploratory review. Vision Development and Rehabilitation 3, 10-22 (2017).

28. Braddick, O., Atkinson, J. \& Wattam-Bell, J. Normal and anomalous development of visual motion processing: motion coherence and 'dorsal-stream vulnerability'. Neuropsychologia41, 1769-1784 (2003).

29. Pavlova, M., Staudt, M., Sokolov, A., Birbaumer, N. \& Krageloh-Mann, I. Perception and production of biological movement in patients with early periventricular brain lesions. Brain126, 692-701 (2003).

30. Weinstein, J. M. et al. Defective motion processing in children with cerebral visual impairment due to periventricular white matter damage. Dev Med Child Neuro/54, e1-8, doi:10.1111/j.1469-

8749.2010.03874.x (2012).

31. Kooiker, M. J., Pel, J. J., van der Steen-Kant, S. P. \& van der Steen, J. A Method to Quantify Visual Information Processing in Children Using Eye Tracking. J Vis Exp, doi:10.3791/54031 (2016).

32. Kooiker, M. J. et al. Quantification of visual function assessment using remote eye tracking in children: validity and applicability. Acta Ophthalmo/94, 599-608, doi:10.1111/aos.13038 (2016).

33. Haxby, J. V. et al. Dissociation of object and spatial visual processing pathways in human extrastriate cortex. Proc Natl Acad Sci U S A88, 1621-1625 (1991).

34. Good, W. V., Jan, J. E., Burden, S. K., Skoczenski, A. \& Candy, R. Recent advances in cortical visual impairment. Dev Med Child Neuro/43, 56-60 (2001).

35. Skoczenski, A. M. \& Norcia, A. M. Development of VEP Vernier acuity and grating acuity in human infants. Invest Ophthalmol Vis Sci40, 2411-2417 (1999).

36. Watson, T., Orel-Bixler, D. \& Haegerstrom-Portnoy, G. Early visual-evoked potential acuity and future behavioral acuity in cortical visual impairment. Optom Vis Sci87, 80-86, 
doi:10.1097/OPX.0b013e3181c75184 (2010).

37. VerMaas, J. R. et al. Beyond the eye: Cortical differences in primary visual processing in children with cerebral palsy. Neuroimage Clin27, 102318, doi:10.1016/j.nicl.2020.102318 (2020).

38. Jensen, O. \& Mazaheri, A. Shaping functional architecture by oscillatory alpha activity: gating by inhibition. Front Hum Neurosci4, 186, doi:10.3389/fnhum.2010.00186 (2010).

39. Bollimunta, A., Chen, Y., Schroeder, C. E. \& Ding, M. Neuronal mechanisms of cortical alpha oscillations in awake-behaving macaques. Journal of Neuroscience28, 9976-9988 (2008).

40. Da Silva, F. L. \& Van Leeuwen, W. S. The cortical source of the alpha rhythm. Neuroscience letters6, 237-241 (1977).

41. Dougherty, K., Cox, M. A., Ninomiya, T., Leopold, D. A. \& Maier, A. Ongoing alpha activity in V1 regulates visually driven spiking responses. Cerebral Cortex27, 1113-1124 (2017).

42. Spaak, E., Bonnefond, M., Maier, A., Leopold, D. A. \& Jensen, O. Layer-specific entrainment of gammaband neural activity by the alpha rhythm in monkey visual cortex. Current Biology22, 2313-2318 (2012).

43. Hughes, S. W. \& Crunelli, V. Thalamic mechanisms of EEG alpha rhythms and their pathological implications. The Neuroscientist11, 357-372 (2005).

44. Lopes da Silva, F. H., Vos, J., Mooibroek, J. \& Rotterdam, A. v. Relative contributions of intracortical and thalamo-cortical processes in the generation of alpha rhythms, revealed by partial coherence analysis. Electroencephalography and clinical neurophysiology50, 449-456 (1980).

45. Saalmann, Y. B., Pinsk, M. A., Wang, L., Li, X. \& Kastner, S. The pulvinar regulates information transmission between cortical areas based on attention demands. Science337, 753-756 (2012).

46. Kahana, M. J., Sekuler, R., Caplan, J. B., Kirschen, M. \& Madsen, J. R. Human theta oscillations exhibit task dependence during virtual maze navigation. Nature399, 781-784 (1999).

47. Bennett, C. R. et al. Virtual Reality Based Assessment of Static Object Visual Search in Ocular Compared to Cerebral Visual Impairment. HC/8, 28-38 (2018).

48. Bottari, D. et al. EEG frequency-tagging demonstrates increased left hemispheric involvement and crossmodal plasticity for face processing in congenitally deaf signers. Neurolmage223, 117315 (2020).

49. Stropahl, M., Bauer, A.-K. R., Debener, S. \& Bleichner, M. G. Source-Modeling auditory processes of EEG data using EEGLAB and brainstorm. Frontiers in neuroscience12, 309 (2018).

50. Delorme, A. \& Makeig, S. EEGLAB: an open source toolbox for analysis of single-trial EEG dynamics including independent component analysis. Journal of neuroscience methods134, 9-21 (2004).

51. Widmann, A., Schröger, E. \& Maess, B. Digital filter design for electrophysiological data-a practical approach. Journal of neuroscience methods250, 34-46 (2015).

52. Delorme, A., Sejnowski, T. \& Makeig, S. Enhanced detection of artifacts in EEG data using higherorder statistics and independent component analysis. Neuroimage34, 1443-1449 (2007). 
53. Bell, A. J. \& Sejnowski, T. J. An information-maximization approach to blind separation and blind deconvolution. Neural computation7, 1129-1159 (1995).

54. Jung, T. P. et al. Removing electroencephalographic artifacts by blind source separation. Psychophysiology37, 163-178 (2000).

55. Jung, T.-P. et al. Removal of eye activity artifacts from visual event-related potentials in normal and clinical subjects. Clinical Neurophysiology111, 1745-1758 (2000).

56. Viola, F. C. et al. Semi-automatic identification of independent components representing EEG artifact. Clinical Neurophysiology120, 868-877 (2009).

57. Oostenveld, R., Fries, P., Maris, E. \& Schoffelen, J.-M. FieldTrip: open source software for advanced analysis of MEG, EEG, and invasive electrophysiological data. Computational intelligence and neuroscience2011 (2011).

58. Maris, E. \& Oostenveld, R. Nonparametric statistical testing of EEG-and MEG-data. Journal of neuroscience methods164, 177-190 (2007).

59. Dale, A. M., Fischl, B. \& Sereno, M. I. Cortical surface-based analysis: I. Segmentation and surface reconstruction. Neuroimage9, 179-194 (1999).

60. Fischl, B. et al. Whole brain segmentation: automated labeling of neuroanatomical structures in the human brain. Neuron33, 341-355 (2002).

61. Fischl, B., Sereno, M. I. \& Dale, A. M. Cortical surface-based analysis: Il: inflation, flattening, and a surface-based coordinate system. Neuroimage9, 195-207 (1999).

62. Fischl, B. et al. Automatically parcellating the human cerebral cortex. Cerebral cortex14, 11-22 (2004).

63. Iglesias, J. E. et al. A probabilistic atlas of the human thalamic nuclei combining ex vivo MRI and histology. Neuroimage183, 314-326 (2018).

64. Keller, S. S. et al. Volume estimation of the thalamus using freesurfer and stereology: consistency between methods. Neuroinformatics10, 341-350 (2012).

65. Niemann, K., Mennicken, V., Jeanmonod, D. \& Morel, A. The Morel stereotactic atlas of the human thalamus: atlas-to-MR registration of internally consistent canonical model. Neuroimage12, 601-616 (2000).

\section{Tables}

\section{Table 1. CVI Participant Demographics}




\begin{tabular}{|c|c|c|c|c|c|c|}
\hline $\begin{array}{l}\text { Subject } \\
\text { ID }\end{array}$ & Sex & Age & $\begin{array}{l}\text { Diagnosis/Associated } \\
\text { Cause of CVI }\end{array}$ & $\begin{array}{l}\text { Preterm } \\
\text { (<32 } \\
\text { weeks)/term }\end{array}$ & $\begin{array}{l}\text { Distance } \\
\text { Visual acuity } \\
\text { RE - LE } \\
\text { (Snellen } \\
\text { equivalent) }\end{array}$ & $\begin{array}{l}\text { Distance } \\
\text { Visual acuity } \\
\text { RE-LE } \\
\text { (logMAR } \\
\text { equivalent) }\end{array}$ \\
\hline 1 & Female & 15 & $\begin{array}{l}\text { birth complication, } \\
\text { global developmental } \\
\text { delay }\end{array}$ & term & $\begin{array}{l}20 / 30- \\
20 / 30\end{array}$ & $0.2-0.2$ \\
\hline 2 & Female & 17 & meningitis, infarct & term & $\begin{array}{l}20 / 50- \\
20 / 60\end{array}$ & $0.4-0.5$ \\
\hline 3 & Male & 19 & $\begin{array}{l}\text { decreased placental } \\
\text { perfusion }\end{array}$ & preterm & $\begin{array}{l}20 / 25- \\
20 / 25\end{array}$ & $0.1-0.1$ \\
\hline 4 & Female & 21 & $\begin{array}{l}\text { periventricular } \\
\text { leukomalacia }\end{array}$ & preterm & $\begin{array}{l}20 / 50- \\
20 / 40\end{array}$ & $0.4-0.3$ \\
\hline 5 & Male & 19 & $\begin{array}{l}\text { focal cortical atrophy, } \\
\text { seizure disorder }\end{array}$ & term & $\begin{array}{l}20 / 60- \\
20 / 60\end{array}$ & $0.5-0.5$ \\
\hline 6 & Male & 15 & genetic & term & $\begin{array}{l}20 / 20- \\
20 / 20\end{array}$ & $0.0-0.0$ \\
\hline 7 & Male & 16 & infection & term & $\begin{array}{l}20 / 25- \\
20 / 25\end{array}$ & $0.1-0.1$ \\
\hline 8 & Male & 22 & $\begin{array}{l}\text { periventricular } \\
\text { leukomalacia }\end{array}$ & preterm & $\begin{array}{l}20 / 40- \\
20 / 25\end{array}$ & $0.3-0.1$ \\
\hline
\end{tabular}

Figures 
A

Success Rate

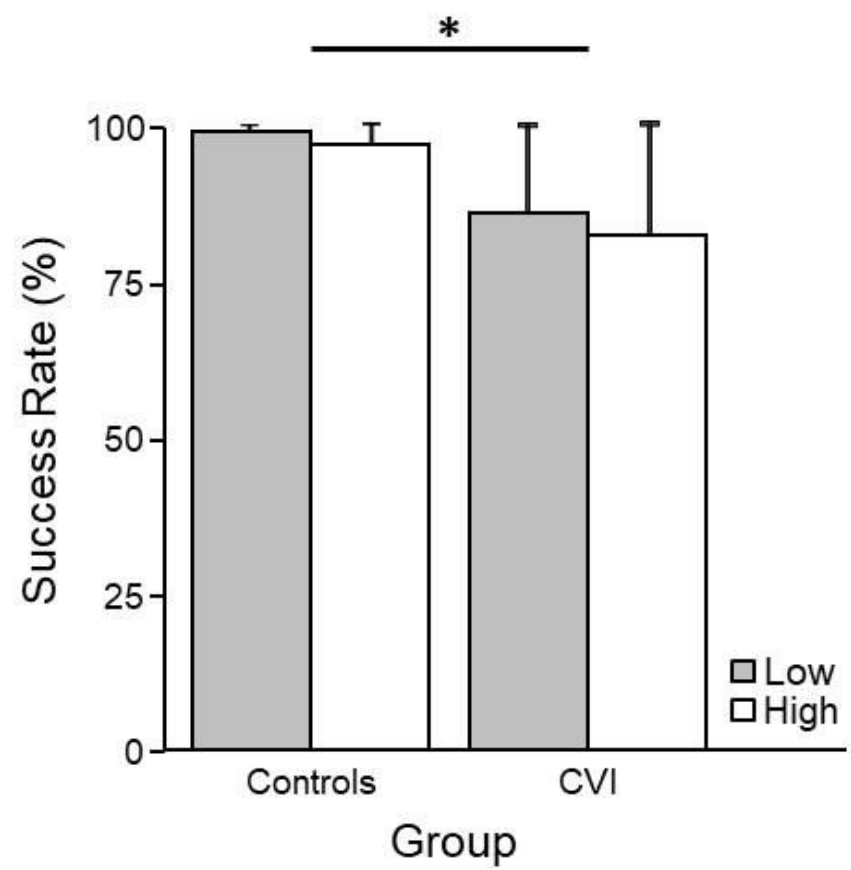

B

Reaction Time

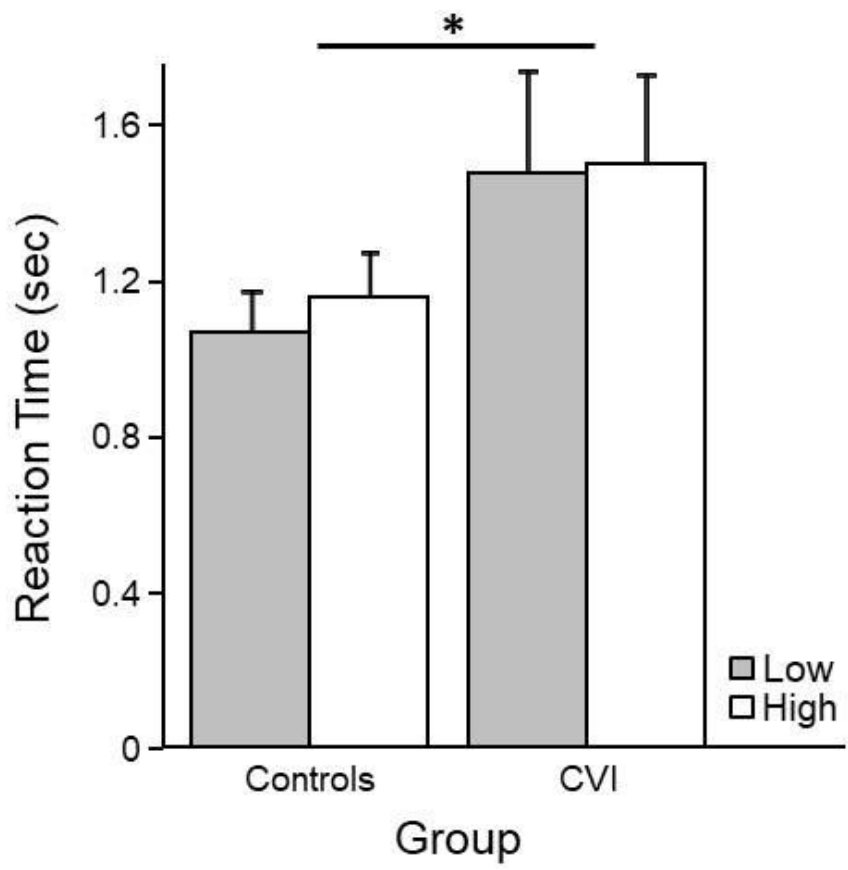

Figure 1

Visual Search Performance. Success rate (A) and reaction time (B) shown separated by task difficulty condition (low and high). For both behavioral outcome measures, significant differences were observed between groups with CVI participants showing impaired performance overall compared to controls (error bars: $\pm S D)$. 
A

Success Rate

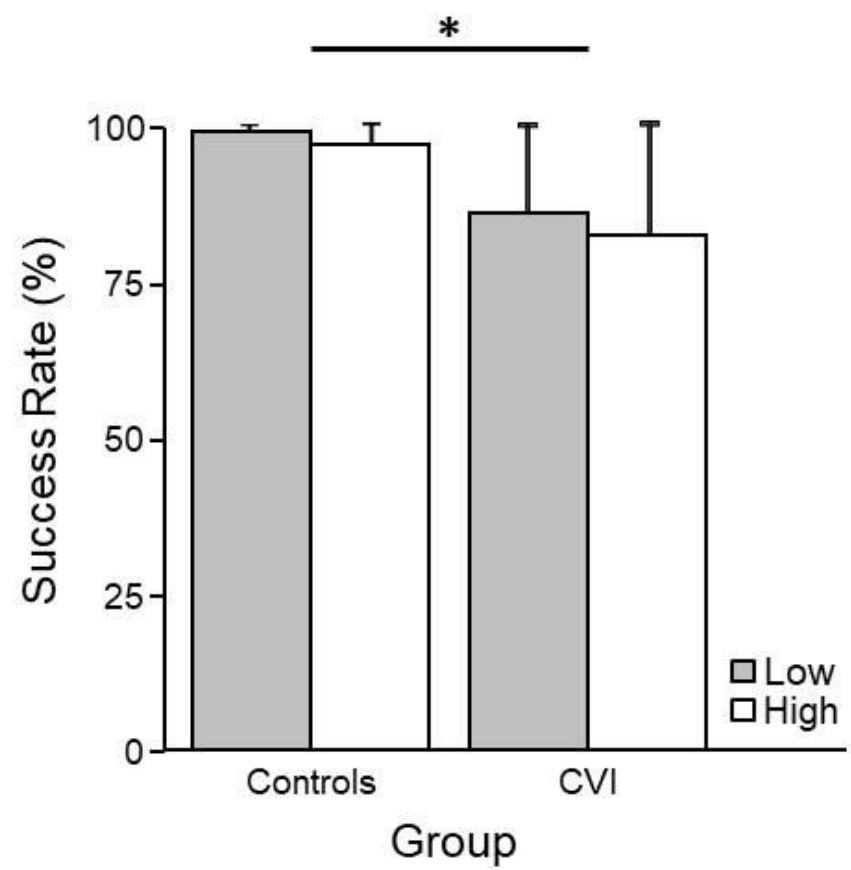

B

Reaction Time

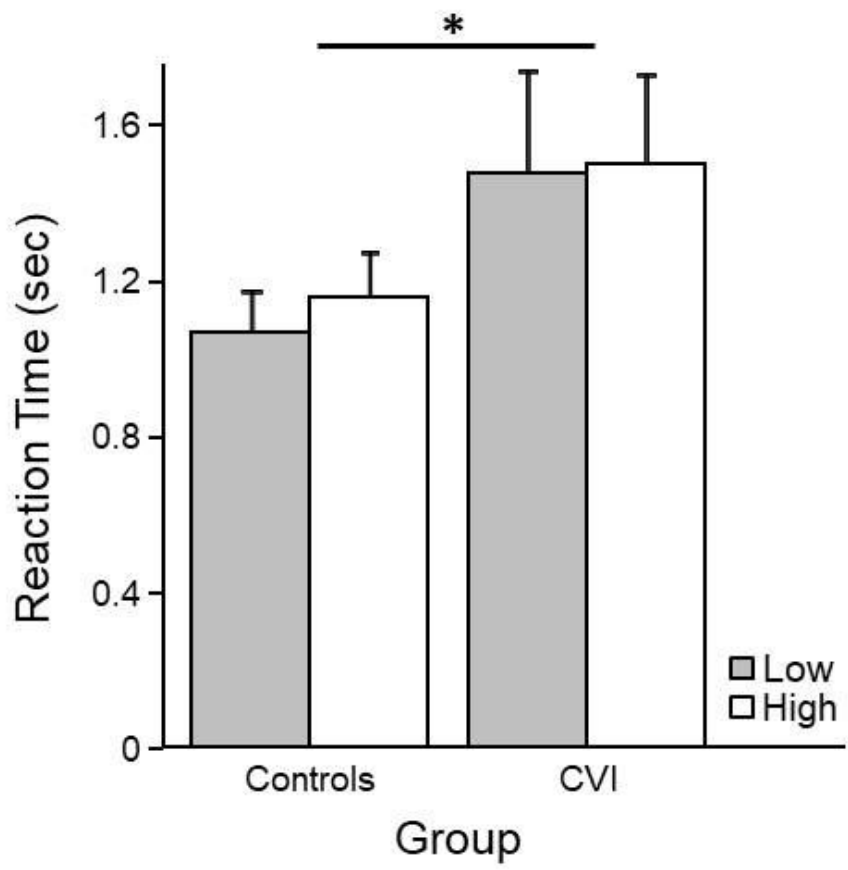

Figure 1

Visual Search Performance. Success rate (A) and reaction time (B) shown separated by task difficulty condition (low and high). For both behavioral outcome measures, significant differences were observed between groups with CVI participants showing impaired performance overall compared to controls (error bars: $\pm S D)$. 

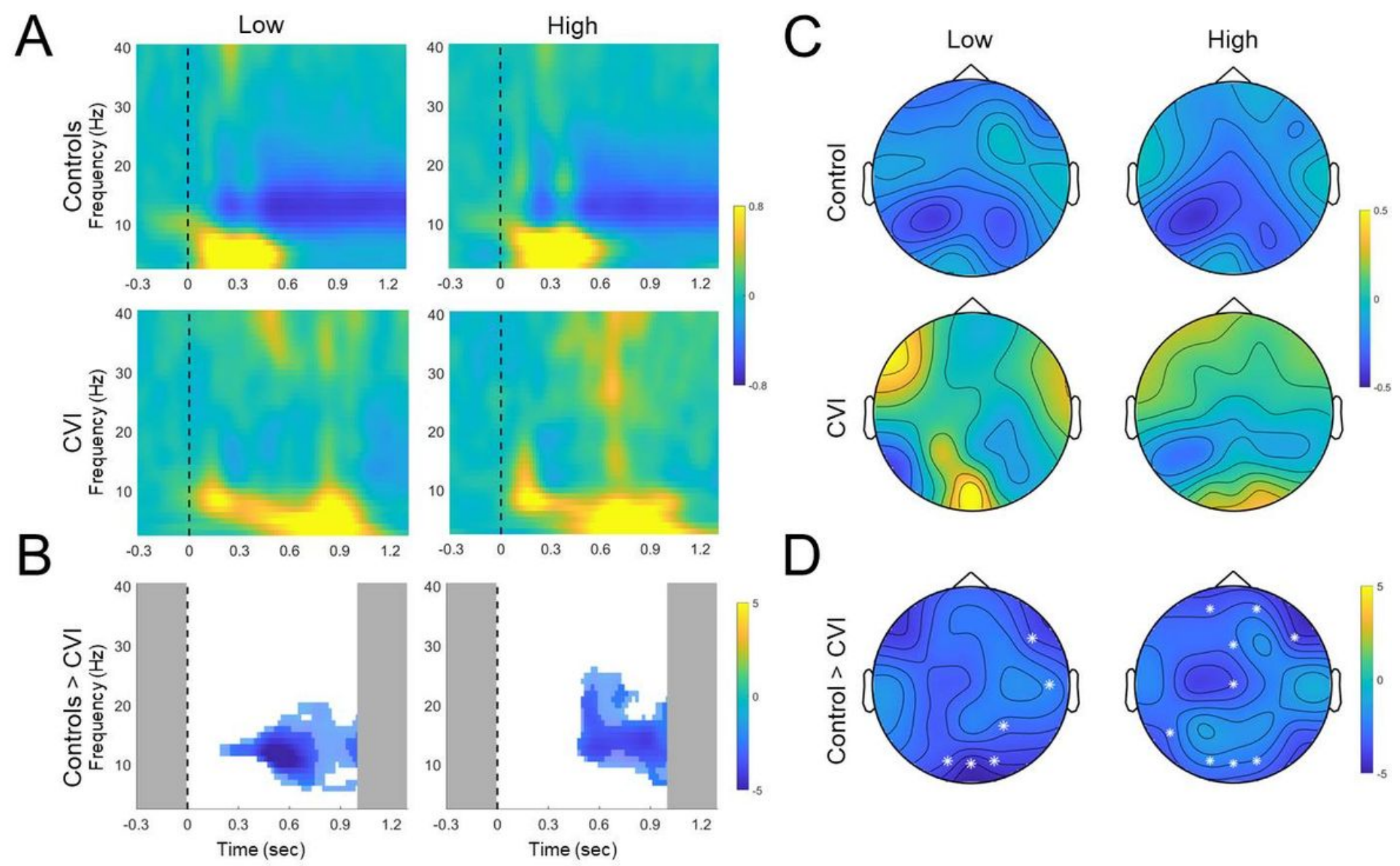

\section{Figure 2}

Time Frequency Plots of Alpha Oscillatory Activity. (A) Relative power signal changes shown in time frequency representations with respect to baseline averaged across the occipital channels $(01, \mathrm{Oz}$, and 02). Data are shown across the frequency range of 3 to $40 \mathrm{~Hz}$, and for a time window of $0.3 \mathrm{sec}$ pre stimulus to $1.2 \mathrm{sec}$ post stimulus. Data for each group (CVI and controls) and task difficulty condition (low and high) are shown separately. Dotted black line represents stimulus onset. Group differences in alpha desynchronization reveal strong and consistent levels of alpha desynchronization in controls (for both task conditions) that are absent in CVI participants. (B) Cluster-based permutation statistical comparison between groups (Controls $>\mathrm{CVI}$ ) separated by task condition. Statistical comparison was performed within a 0 to $1 \mathrm{sec}$ post-stimulus time window across the frequency range of 3 to $40 \mathrm{~Hz}$ and all electrodes. Significant differences are observed between groups in the alpha range and in particular, within the peak window. (C) Scalp topographies of the baseline corrected alpha oscillatory activity shown for the time windows in which group comparisons revealed highest statistical differences ( 0.440 to 0.500 sec for low and 0.500 to 0.560 sec for high, post-stimulus). Data for each group (control and CVI) and task difficulty condition (low and high) are shown separately. Group differences can be observed at the whole scalp level and in particular, across the posterior electrode locations. (D) Scalp topographies of the cluster-based permutation results performed between groups for each condition (statistically significant channels highlighted with a white asterisks). The most robust differences emerged for the low task difficulty condition at the occipital electrodes. 

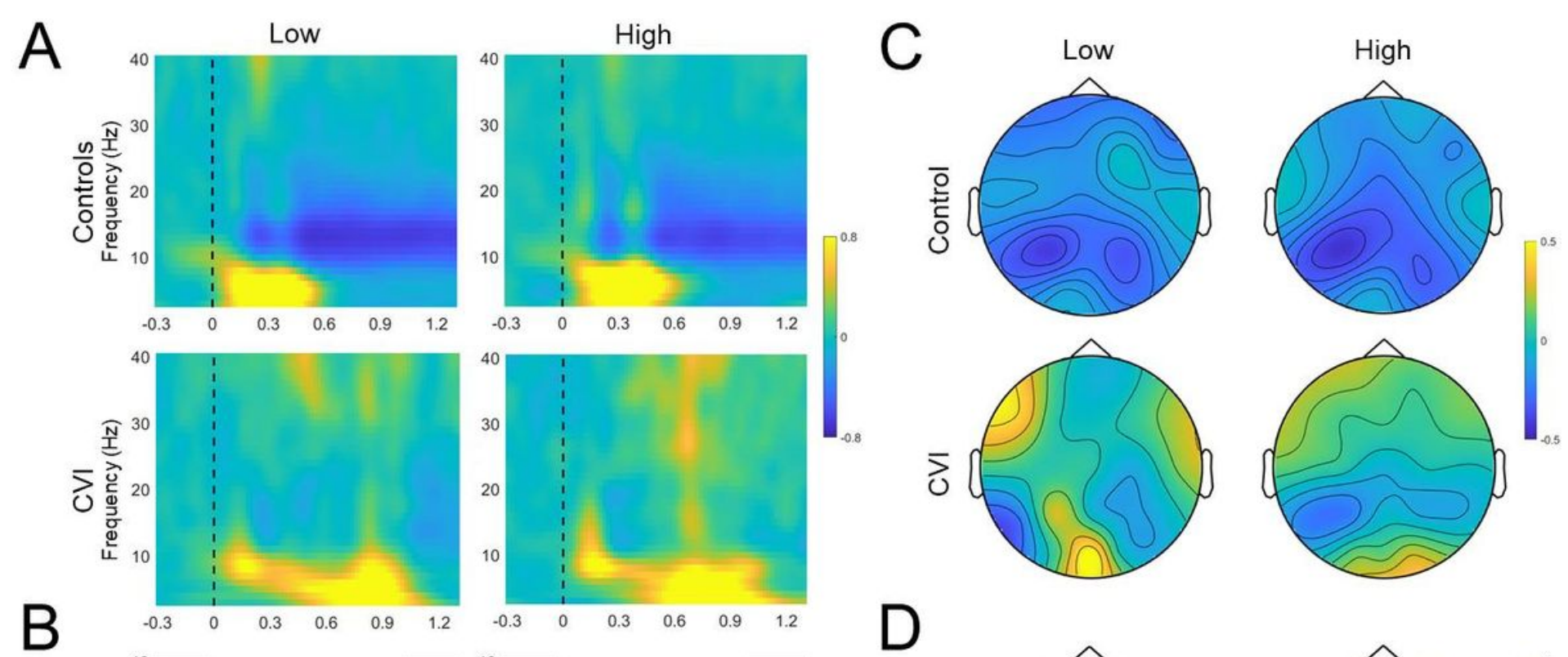

$\mathrm{D}$
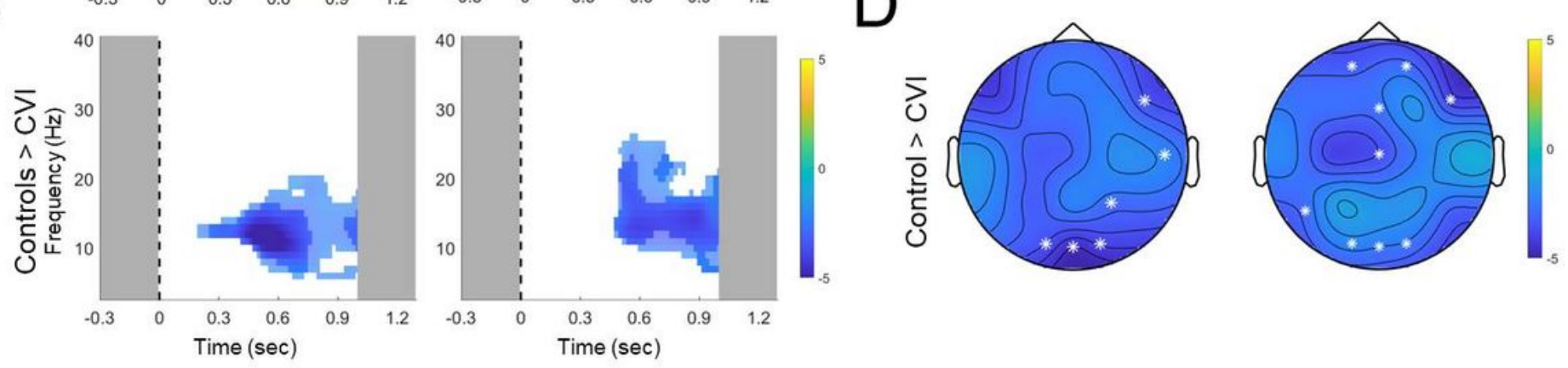

\section{Figure 2}

Time Frequency Plots of Alpha Oscillatory Activity. (A) Relative power signal changes shown in time frequency representations with respect to baseline averaged across the occipital channels $(01,0 z$, and 02). Data are shown across the frequency range of 3 to $40 \mathrm{~Hz}$, and for a time window of $0.3 \mathrm{sec}$ pre stimulus to $1.2 \mathrm{sec}$ post stimulus. Data for each group (CVI and controls) and task difficulty condition (low and high) are shown separately. Dotted black line represents stimulus onset. Group differences in alpha desynchronization reveal strong and consistent levels of alpha desynchronization in controls (for both task conditions) that are absent in CVI participants. (B) Cluster-based permutation statistical comparison between groups (Controls $>\mathrm{CVI}$ ) separated by task condition. Statistical comparison was performed within a 0 to $1 \mathrm{sec}$ post-stimulus time window across the frequency range of 3 to $40 \mathrm{~Hz}$ and all electrodes. Significant differences are observed between groups in the alpha range and in particular, within the peak window. (C) Scalp topographies of the baseline corrected alpha oscillatory activity shown for the time windows in which group comparisons revealed highest statistical differences ( 0.440 to 0.500 sec for low and 0.500 to 0.560 sec for high, post-stimulus). Data for each group (control and CVI) and task difficulty condition (low and high) are shown separately. Group differences can be observed at the whole scalp level and in particular, across the posterior electrode locations. (D) Scalp topographies of the cluster-based permutation results performed between groups for each condition (statistically significant channels highlighted with a white asterisks). The most robust differences emerged for the low task difficulty condition at the occipital electrodes. 

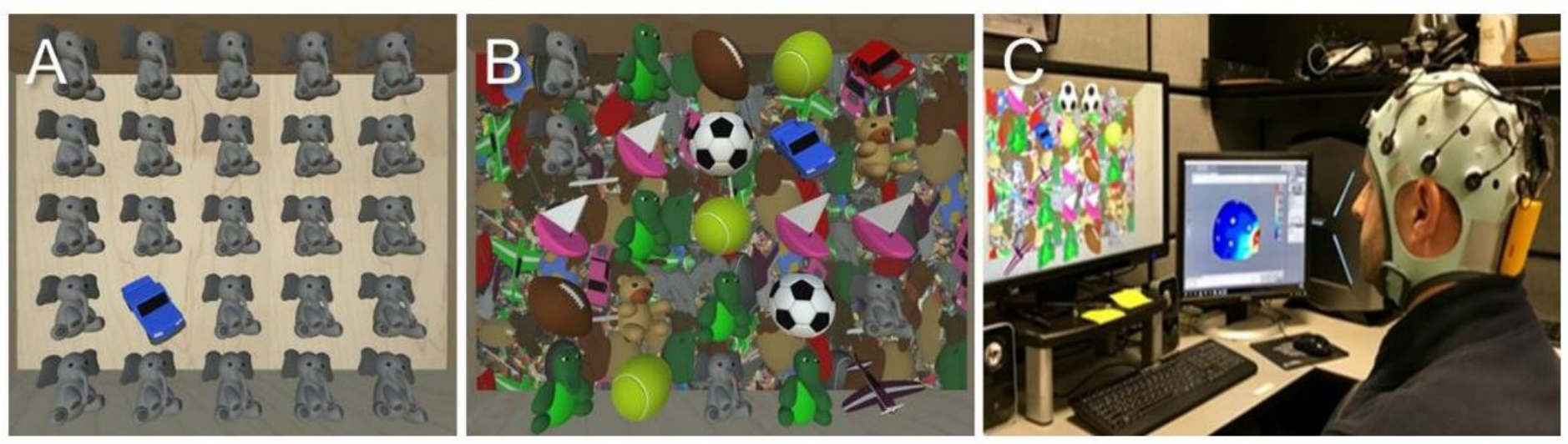

\section{Figure 3}

Experimental Setup. The "virtual toy box" visual search task showing the (A) low and (B) high task difficulty conditions. The participant is instructed to search for the pre-selected target (in the case, a blue truck) on each trial. (C) Visual search task combined with wireless EEG recording montage (Neuroelectrics Enobio 20 channel Wireless EEG Recording System).
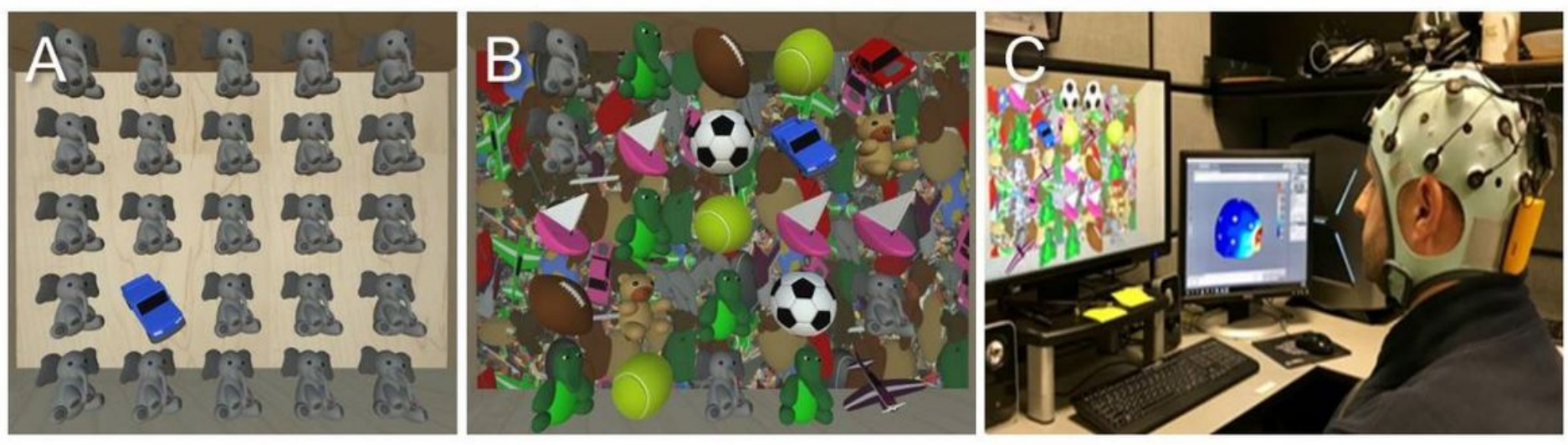

\section{Figure 3}

Experimental Setup. The "virtual toy box" visual search task showing the (A) low and (B) high task difficulty conditions. The participant is instructed to search for the pre-selected target (in the case, a blue truck) on each trial. (C) Visual search task combined with wireless EEG recording montage (Neuroelectrics Enobio 20 channel Wireless EEG Recording System).

\section{Supplementary Files}

This is a list of supplementary files associated with this preprint. Click to download.

- SupplementalFigure1.png

- SupplementalFigure1.png 
- Supplementalmaterialsfigure1.pdf

- Supplementalmaterialsfigure1.pdf 